Article

\title{
Energy Management with Support of PV Partial Shading Modelling in Micro Grid Environments
}

\author{
Marco Severini *, Emanuele Principi, Marco Fagiani, Stefano Squartini and Francesco Piazza \\ Department of Information Engineering, Università Politecnica delle Marche, Via Brecce Bianche 1, \\ 60131 Ancona, Italy; e.principi@univpm.it (E.P.); m.fagiani@univpm.it (M.F.); s.squartini@univpm.it (S.S.); \\ f.piazza@univpm.it (F.P.) \\ * Correspondence: m.severini@univpm.it; Tel.: +39-071-220-4128
}

Academic Editor: Peter J. S. Foot

Received: 19 February 2017; Accepted: 24 March 2017; Published: 1 April 2017

\begin{abstract}
Although photovoltaic power plants are suitable local energy sources in Micro Grid environments, when large plants are involved, partial shading and inaccurate modelling of the plant can affect both the design of the Micro Grid as well as the energy management process that allows for lowering the overall Micro Grid demand towards the main grid. To investigate the issue, a Photovoltaic Plant simulation model, based on a real life power plant, and an energy management system, based on a real life Micro Grid environment, have been integrated to evaluate the performance of a Micro Grid under partial shading conditions. Using a baseline energy production model as a reference, the energy demand of the Micro Grid has been computed in sunny and partial shading conditions. The experiments reveal that an estimation based on a simplified PV model can exceed by $65 \%$ the actual production. With regards to Micro Grid design, on sunny days, the expected costs, based on a simplified PV model, can be 5.5\% lower than the cost based on the double inverter model. In single cloud scenarios, the underrating can reach $28.3 \%$. With regard to the management process, if the energy yield is estimated by means of a simplified PV model, the actual cost can be from $17.1 \%$ to $21.5 \%$ higher than the theoretical cost expected at design time.
\end{abstract}

Keywords: micro grid; mixed integer linear programming; energy management; renewable energy sources; partial shading; photovoltaic systems

\section{Introduction}

The limited availability of fossil fuels, as well as the limitation of present day electrical energy distribution paradigms, have led to the adoption of new distribution approaches such as Micro Grid paired with localized energy production, in most cases based on renewable energy sources.

Although several energy production technologies can be adopted, such as fuel cell power generators [1,2], combined heat and power plants [3,4], wind micro turbine [5,6], or a combination of several technologies [7], photovoltaic energy production plants are the most commonly used. Reduced maintenance requirements, absence of production costs and pollution, be it either $\mathrm{CO}_{2}$ emissions or noise, are among the strong points of photovoltaic solutions. Nonetheless, even though the benefits of the photovoltaic technology steadily promote the adoption of solar plants, several shortcomings are to be addressed, in order to optimize the energy yield and overcome some limitations of this technology. Differently from other renewable energy based technologies, in fact, the photovoltaic production does not depend only on the energy availability of the source, that is the mean irradiation level, but it relates also to the degree of homogeneity of the irradiation over the plant [8-11].

In fact, since a photovoltaic plant is a large array of photovoltaic cells [12], whose output shows a non-linear Power-Voltage (P-V) characteristic and one maximum power point (MPP), the interaction among the cells may limit the overall power output of the plant. In this case, MPP tracking (MPPT) 
technologies may play an important role [13-16] in the plant performance. However, if the plant occupies a large area, partial shading of the plant may occur, thus disrupting the activity of the MPPT algorithm.

In this case, the design of the plant topology represents one of the key factors to limit the effects of partial shading, although the improvement of the system performance cannot disregard the balance between construction costs and maintenance costs. To investigate this problem, a software framework has been developed by the authors to simulate the behaviour of a real life PV plant and evaluate its performance under various weather conditions [17].

In a Micro Grid environment, however, when an energy management system is used, a power plant is an integral part of the system [18], rather than a separate entity, in that its performance can affect the efficiency of the energy management. In fact, if energy management systems are deployed, the ability to correctly predict the plant behaviour, in order to assess the hourly energy production, is required in order to optimally allocate the resources.

For instance, although a crude estimate of the production can be used, based on the solar irradiation forecasts [19], a detailed modelling that takes into account not only the plant behaviour but also the weather forecasts can allow a better assessment of the production so that the energy yield is not overestimated.

On purpose, in this work, a software framework that simulates the energy output of a configurable PV system is proposed to evaluate the system performance in different conditions. Indeed, although many PV Plant design tools are available, such as the System Advisor Model provided by NREL [20], in most cases, they are aimed at commercial applications rather than research; thus, they model commercially available devices, and rarely provide the means to implement and investigate specific aspects of the PV plant such as the MPPT algorithm. In order to overcome this limitation, a simulation framework has been devised.

The framework is able to take into account weather conditions, solar irradiations, partial shading, the type and arrangement of PV modules, and implements the Perturb and Observe (P\&O) $[13,14,21]$ and the Incremental Conductance (IC) [15,16,22-24] MPPT tracking algorithms both with and without Global Peak Tracking (GPT) [25]. The framework has been integrated into a residential and Micro Grid management framework [26-28], in providing energy yield forecasts, to plan ahead the use of the energy storage subsystems. The framework can be used both as a design tool to properly gauge the power required from the plant and as a forecasting tool to support the energy management. The integrated framework is also available upon request.

Two case studies have been considered in the evaluation: the simulation of a real life power plant and the simulation of an energy management system. The power plant has a total surface of about $6700 \mathrm{~m}^{2}$ and a nominal power output of about $1000 \mathrm{kWp}$. In the energy management case study, a real life Micro Grid has been considered, with a district composed of 44 buildings. The simulation has been performed with different irradiation conditions, topologies, and MPPT algorithms. The results show that, by using the proposed framework, it is possible to properly estimate the cost estimation in the design of a Micro Grid.

The outline follows. In Section 2, the elements at the base of the PV model are presented, whereas Section 3 introduces the energy management framework. In Section 4, we describe the simulation scenario and analyse the results for a case of study. Section 5 draws the conclusions.

\section{Photovoltaic Plant Simulating Model}

This section focuses on the photovoltaic plant simulation model, in order to explain how the structure of the PV array and the pattern of irradiation with dynamic shading have been implemented.

\subsection{Circuital Model}

The energy production of the PV modules has been modelled based on the I-V characteristic of the PV cell. On purpose, in order to better evaluate the effects of partial shading and varying solar 
irradiation levels, a double diode PV model has been used, since this modelling approach is able to describe the behaviour of a PV cell more accurately [29]. The implemented equation is an evolved form of the single diode PV cell model [30,31], in that it models the current loss due to the recombination of charge carriers through two diodes [32-34]. The equation is reported below:

$$
I=I_{P V}-I_{01} \cdot\left[e^{\frac{q\left(V+I \cdot R_{S}\right)}{k \cdot T_{C} \cdot m_{1}}}-1\right]-I_{02} \cdot\left[e^{\frac{q\left(V+I R_{S}\right)}{k \cdot T_{C} \cdot m_{2}}}-1\right]-\frac{\left(V+I \cdot R_{S}\right)}{R_{P}},
$$

where $I$ is the output current, $I_{P V}$ is the current generated by the incident light, whereas $I_{01}$ and $I_{02}$ are the reverse saturation currents of the diodes. The term $k T / q$ is the thermal voltage of the P-N junction, where $q$ is the charge of the electron, $k$ is the Boltzmann's factor and $T$ the temperature of the junction (in Kelvin). Additionally, the terms $m_{1}$ and $m_{2}$ are the ideality factors of the two diodes, $R_{S}$ is the series resistance and $R_{P}$ is the parallel resistance. Thus, the quantity $\left(V+I \cdot R_{S}\right) / R_{P}$ is the shunt current loss.

According to [32-34], the parallel resistance $R_{P}$ has been modelled as follows:

$$
R_{P}=\frac{\left(V_{m p}+I_{m p} \cdot R_{S}\right)}{I_{P V}-I_{0}\left[e^{\frac{V_{m p}+I_{m p} R_{S}}{V_{T}}}+e^{\frac{V_{m p}+I_{m p} R_{S}}{(p-1) V_{T}}}-2\right]-\frac{P_{m p}}{V_{m p}}},
$$

where the quantities $V_{m p}$ and $I_{m p}$ represent, respectively, the voltage and the current at the maximum power point, and $V_{T}$ is the thermal voltage of the P-N junction. In the framework, $R_{P}$ and $R_{S}$ are calculated iteratively [34] and $m_{1}$, the ideality factor of the first diode, has been set to 1 , whereas $m_{2}$, the ideality factor of the second diode, has been set to 1.2 and $p$ has been chosen so that $\left(m_{1}+m_{2}\right) / p$ is equal to 1 [30]. Moreover, the implementation has been validated against the manufacturer's specifications of a real life PV panel.

The model of each PV module takes into account the actual number of PV cells of the panel, and PV arrays are modelled as strings of PV modules each equipped with a bypass diode connected in anti-parallel.

Indeed, a PV module is composed of cells connected in series in order to obtain an open circuit voltage that is sufficiently high for power production: connecting $N$ cells, the total open circuit voltage is $N$ times the one of a single cell, while the short circuit current is the same for every cell [34]. Additionally, a bypass diode is paired to every cell so that a single cell cannot limit the short circuit current in partial shading conditions. In this way, the current is not limited, but the P-V characteristic of the module now can exhibit multiple local maxima.

Usually, power plants are composed of several PV modules connected in series that form a module string with the desired voltage output. In turn, several strings are connected in parallel in order to obtain the desired current output. In conditions of partial shading, every string can be considered as an independent subsystem, whereas the current output of the array is the sum of the currents of the strings [8].

In the framework, bypass diodes are supposed ideal, i.e., their power losses are not considered and their effect has been approximated by simply disconnecting the modules affected by shadowing. Thus, the characteristic curves of the plant have been computed as the sum of the curves of the strings. The curves of the strings, in turn, have been computed as the sum of the curves of as many panel sub sets as many irradiation levels are present over a string.

Indeed, as shown in Figure 1a, considering three irradiation levels over a single string of the PV model, the characteristic curve shown in Figure $1 b$ is the result of the three distinct curves shown in Figure $2 \mathrm{~d}$. These represent the three irradiation scenarios depicted in Figure 2a-c: in Figure $2 \mathrm{c}$, all the modules are able to supply energy, since the drawn power level is below or equal to the output power of the least irradiated panels. In Figure $2 b$, the most shadowed modules are disconnected from the string by the bypass diodes, since the drawn power level rises above the output power of the least irradiated panels and their output voltage drops. In this case, only the remaining modules can supply 
energy. Finally, Figure 2a shows the case where the drawn power level rises again and only the most irradiated modules contribute to the energy production. Therefore, the overall power output in Figure $1 \mathrm{~b}$ is represented by the envelope of the single curves in Figure $2 \mathrm{~d}$.

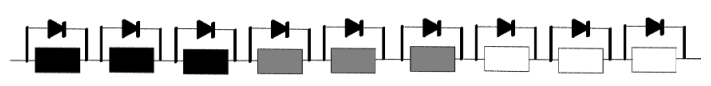

(a)

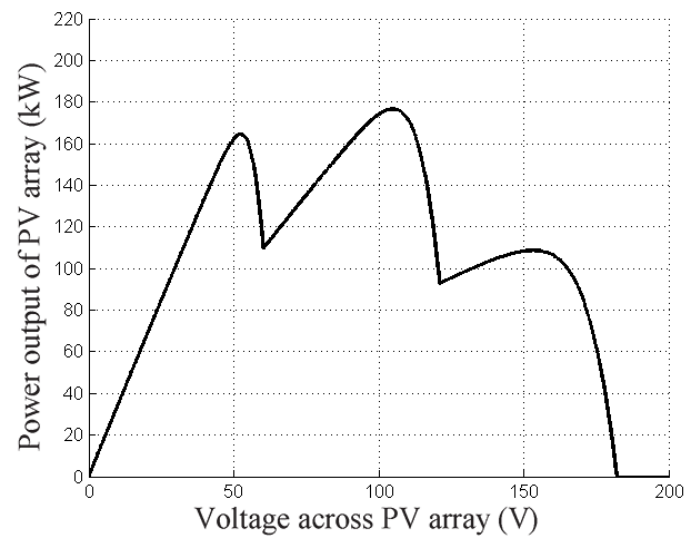

(b)

Figure 1. Panel strings in heterogeneous irradiation conditions: if the PV panels of a string are subject to different irradiation levels (a); the P-V curve of the string (b) shows different local maxima.

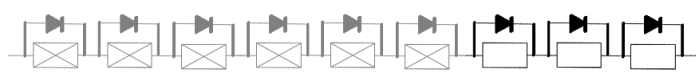

(a)

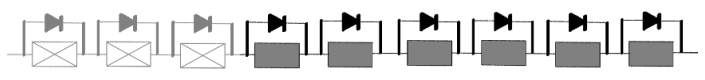

(b)

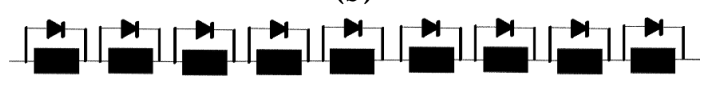

(c)

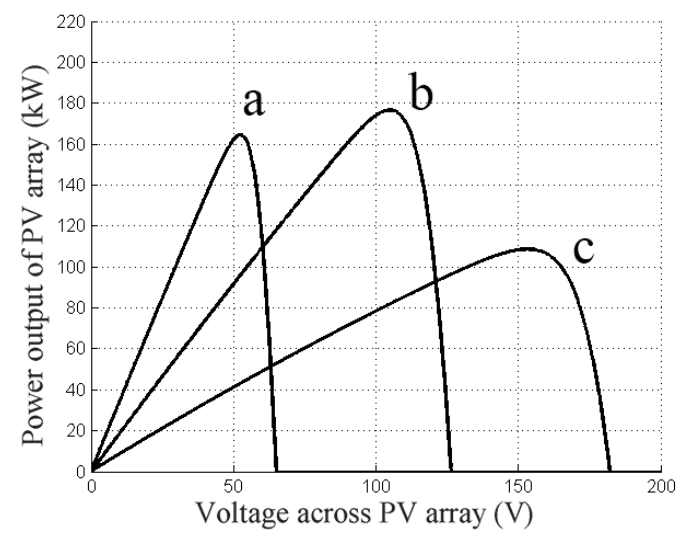

(d)

Figure 2. Overall P-V curve as the sum of P-V curves of homogeneously irradiated panel sub strings: the P-V curve (a) is the output of the most irradiated panels; the P-V curve (b) is equal to the output of a string subject to an intermediate irradiation level, whereas the least irradiated panels are disconnected; the P-V curve (c) is equal to the output of a string subject to the lowest irradiation level; the local maxima appear because, as the output voltage grows, the less irradiated panels begin to operate limiting the power output of the more irradiated ones; (d) Each PV characteristic. 


\subsection{Solar Irradiation and Shading Pattern}

The incident solar power on each panel has been obtained by using its tilt angle, the elevation angle of the sun, and the monthly mean daily global solar irradiation [35]. The time resolution is $1 \mathrm{~min}$, and the geographical coordinates of the town of Ancona (Italy) have been used to take into account the effect of the geographical position of the plant.

The shadowing pattern is represented by a set of matrices, one per minute, whose elements represent a scaling factor for the corresponding PV module. The pattern takes into account the different causes of shadowing of the modules, such as isolated clouds drifting over the PV plant, fog or cloudy weather.

\subsection{Maximum Power Point Tracking}

The output voltage of a PV module depends not only on the incident solar irradiation and the working temperature, but also on the amount of current being drawn. As such, in order to extract the maximum amount of energy from the module, the load has to be controlled so that the working point of the module matches the MPP of its P-V curve. Since the MPP tends to drift depending on irradiation and temperature, a MPP tracking algorithm is required to properly drive the power draw.

In partial shading conditions, the P-V curve of a string of the module can exhibit multiple local maxima as an effect of the bypass diodes. This interferes with MPP tracking algorithms and can reduce the performance of the plant. In the framework, this phenomenon can be studied by means of the implemented MPP tracking algorithms, namely the Perturb and Observe ( $\mathrm{P} \& \mathrm{O})[13,14,21]$, the Incremental Conductance (IC) [15,16,22-24], both with and without the Global Peak Tracking (GPT) in [25].

$\mathrm{P} \& \mathrm{O}$ operates by perturbing the output voltage in the neighbourhood of the working point. This allows for detecting changes in the power output and, consequently, adjusting the system to work at the point that provides greater power output. The IC algorithm calculates the derivative of the current supplied by the system with respect to the output voltage in order to obtain the conductance. The rationale behind IC is that the slope of the P-V curve is null at the MPP, positive at its left and negative at its right. Differently from $\mathrm{P} \& \mathrm{O}$ and IC, the GPT algorithm is able to deal with the multiple local maxima of the P-V curve. The algorithm operates on the principle that the lower the value of a local maximum, the farther it is from the global one. Hence, GPT can move the working point to one local maximum to the global one, detecting the closest maximum by means of a large perturbation. By applying a large perturbation, either on a period-based scheme or on an event-based scheme, every local maximum, rather than the whole P-V curve, is evaluated. Details on the algorithm are shown in Figures 3 and 4 .

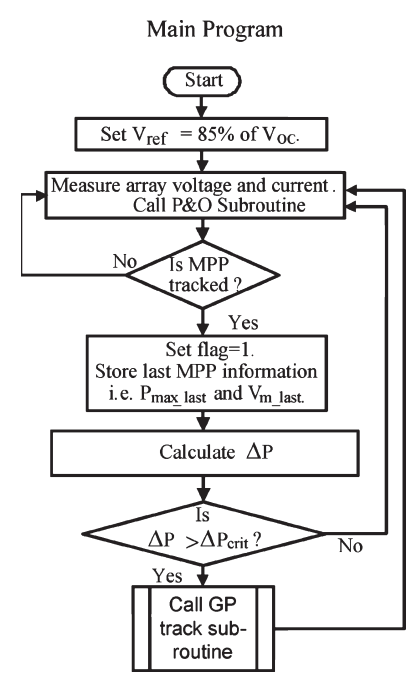

Figure 3. Implementation of the Maximum Power Point (MPP) with support from the Global Peak Tracking approach proposed in [25]. 


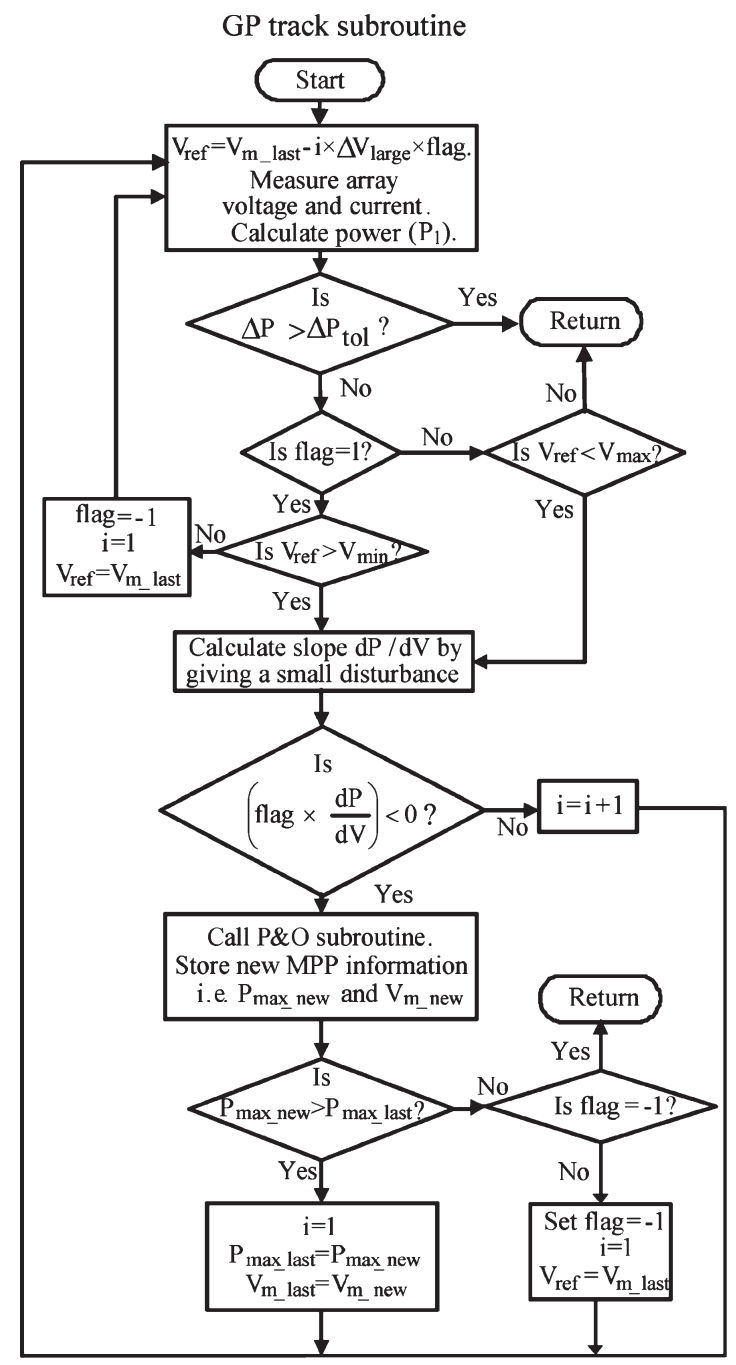

Figure 4. Implementation of the Global Peak Tracking subroutine based on [25].

\section{Residential Environment Real Life Based Model}

This section focuses on the other side on the residential environment simulation model, and focuses on the model that is used to manage both energy and resources within the Micro Grid environment.

\subsection{Reference Environment}

The Micro Grid residential environment, namely the Leaf House, is the reference used to implement the proposed framework. The Leaf House is one of the six international case studies selected by the IEA Task 40/ECBCS Annex 52 “Towards Net Zero Energy Solar Buildings" [36].

By monitoring said environment, a data set has been compiled by collecting data regarding both local demand, of electricity and heat, and local solar based production, of heat and hot water. The integrated subsystems have also been used as a reference to model the various elements of the proposed framework [37]. Many of the details regarding both the environment and the framework are omitted for conciseness sake, but they can be found in our previous work [37].

\subsection{Modelling Approach}

The Micro Grid simulation framework has been implemented to model the energy distribution within the environment. Thus energy production, consumption, storage and transport have been 
modelled. The energy costs, then, have been included to weight the different contributions, as to lower the energy bill, as well as to improve the efficiency of the residential environment.

Since the modelling process has required only linear equations to represent the energy balances, the framework has been implemented as a Mixed Integer Linear Programming (MILP) problem. The MILP problem models the flux of energy among the subsystems within the residential environment. Solving the problem provides the optimal energy management schedule as a result, which leads to an improved environment efficiency.

The behaviour of the system has been modelled as a set of algebraic expressions that describes the balance of energy both within each module and within the environment among the modules.

With respect to the previous works [26,37], the structure of the framework and the simulation algorithm remain unchanged; therefore, those aspects are not discussed in the present manuscript.

The MILP problem assumes the generic form:

Minimize:

$$
\mathbf{f}^{\mathrm{T}} \mathbf{x}
$$

Subject to:

$$
\begin{gathered}
\mathbf{b}_{1} \leq \mathbf{A x} \leq \mathbf{b}_{\mathbf{u}}, \\
\mathbf{l}_{\mathbf{b}} \leq \mathbf{x} \leq \mathbf{u}_{\mathbf{b}},
\end{gathered}
$$

where $\mathbf{f}$ is the coefficient vector of the objective function to be minimized, the matrix $\mathbf{A}$ is the coefficient matrix of the linear constraints, modelled as inequalities, and $\mathbf{x}$ is the vector of the problem variables. Based on this representation, the variables used to model the energy fluxes are recalled:

- $E P_{a}$ : amount of energy purchased to supply the entire structure;

- $E S_{a}$ : amount of energy surplus resulting from the entire structure;

- $E S p_{a}$ : amount of solar thermal energy production;

- $\quad C_{a}$ : amount of energy being stored;

- $\quad E_{p}$ : the energy purchase price;

- $\quad E P_{i}$ : energy required by the $i$-th electrical block with $i=1,2,3$.

The objective function to be minimized has been based on the overall electrical and thermal energy costs. The total energy that has to be purchased, $E P_{a}(t)$, is given by the sum of the purchased energy necessary to supply each block at the $t$-th timeslot:

$$
E P_{a}(t)=E P_{1}(t)+E P_{2}(t)+E P_{3}(t)+E g_{1}(t)+E g_{2}(t)+E P_{h w}(t)+E P_{h e}(t)+E P_{r e}(t) .
$$

The energy surplus $E S_{a}(t)$ is the sum of the surplus provided to each block from the local power plant at the $t$-th timeslot:

$$
E S_{a}(t)=E S_{1}(t)+E S_{2}(t)+E S_{3}(t) .
$$

Therefore, the objective function can be expressed as:

$$
Q=\sum_{t=1}^{24}\left(E P_{a}(t)\right) \cdot E_{p}(t)+E S_{a}(t) \cdot 10^{-6}-E S p_{a}(t) \cdot 10^{-6}+C h_{a}(t) \cdot 10^{-7},
$$

where $E S_{a}$ represents the overall energy surplus to be demoted, $E S p_{a}$ represents the solar thermal energy production to be promoted, and $C h_{a}$ denotes the energy routed towards the storage, which is accounted for to price the activity of the storage to be restricted.

The quantities $10^{-6}$ and $10^{-7}$ are arbitrary values acting as scaling factors. Said values have been introduced to exert additional control over the activity of the system. They have been chosen based on 
the fact that energy is accounted in Wh, and the price $E_{p}$ is accounted in $€$ per Wh, that is, the price of a Wh of purchased energy has an order of magnitude equal to $10^{-5}$.

In fact, since the energy being stored has a value $\left(10^{-7}\right)$, the manager is required to limit the activity of the storage. Similarly, since the energy surplus and the solar thermal energy have a value, the manager is required to account their amount. In fact, on the one hand, removing these amounts from the objective function allows the manager to ignore solar production, leaving the energy unused, or to dissipate the energy surplus through the storage to lower the unused surplus. On the other hand, including said amounts in the objective function while assuming a scaling factor equal to 1 forces the energy manager to minimize both the amount of energy surplus and the storage activity, rather than the energy bill. Thereby, the scaling factors are used to weight the optimization priority of some aspects of the system activity with respect to the management of the energy purchase. As such, they are part of the energy management policy and can be adjusted based on the user needs.

\section{Case Study and Experimental Results}

In the present work, both a photovoltaic power plant and a residential environment have been modelled to implement a framework that can be used either as a design tool or a management tool in a Micro Grid environment. For instance, both the models of the photovoltaic plant and the residential environment have been based on real-life facilities. The software framework is based on MathWorks MatLab R2014b, the Parallel Computing Toolbox 6.5, the OPTI Toolbox version 2.11 [38] and the Solving Constraint Integer Programs (SCIP) 3.0.2 solver. The experiments have been performed on a Windows 8.1 HP Pavilion Laptop, based on an Intel Core i7-4700HQ CPU and with 8 GB of RAM.

\subsection{Power Plant Simulation Case Study}

The simulation model of the photovoltaic power plant has been complemented with data obtained from real-life PV modules. As a reference, a plant based on 4160 CanadianSolar PV panels (model CS6P-240P [39]) has been used. The total surface of the panels amounts to $6691.42 \mathrm{~m}^{2}$, and the nominal output power of the plant amounts to $998.4 \mathrm{kWp}$. The panels are divided in 208 strings of 20 modules each. Moreover, the strings are divided in 13 groups of 16 strings each. The panels face south and with a $30^{\circ}$ tilt angle with respect to the horizon. Shadowing from the panels has been avoided by placing the panels far enough from each other. A simplified sketch of the plant surface is presented in Figure 5 .

The performance of the model has been evaluated on two different irradiation conditions, that is:

(a) sunny day,

(b) single cloud shading.

The sunny day scenario (a) is based on the irradiation profile and weather conditions of 28 August. In this case, a uniform irradiation level across the plant is assumed. The single cloud scenario (b), on the other hand, simulates the shading effect of a cloud that moves across the plant, which results in the irradiation level, over the plant, to not be uniform. In this case, the irradiation profile, used in the sunny day scenario, has been modified to model the effect of partial shading. Regarding the temperature, in order to simplify the analysis and focus on the effect of partial shading, a constant temperature value of about $25^{\circ} \mathrm{C}$ has been used as input in both scenarios.

Based on these irradiation scenarios, three different topologies have been evaluated, along with a baseline configuration, based on a fixed efficiency level for the panels rather than an accurate simulation model:

(1) a centralized set-up has been modelled by assuming a dual inverter for the entire plant (two in total) [40-42];

(2) a macro-block set-up has been modelled by assuming the adoption of one inverter for each subset of panel strings (13 in total);

(3) a micro-block set-up has been modelled by assuming the adoption of one inverter per panel string (208 in total); 
(4) a micro-block baseline set-up has been devised by assuming a fixed $15 \%$ efficiency level without inverter losses.

For instance, the baseline configuration (4) has been proposed since, in literature, a fixed efficiency value is often used to compute the energy production from solar irradiation.

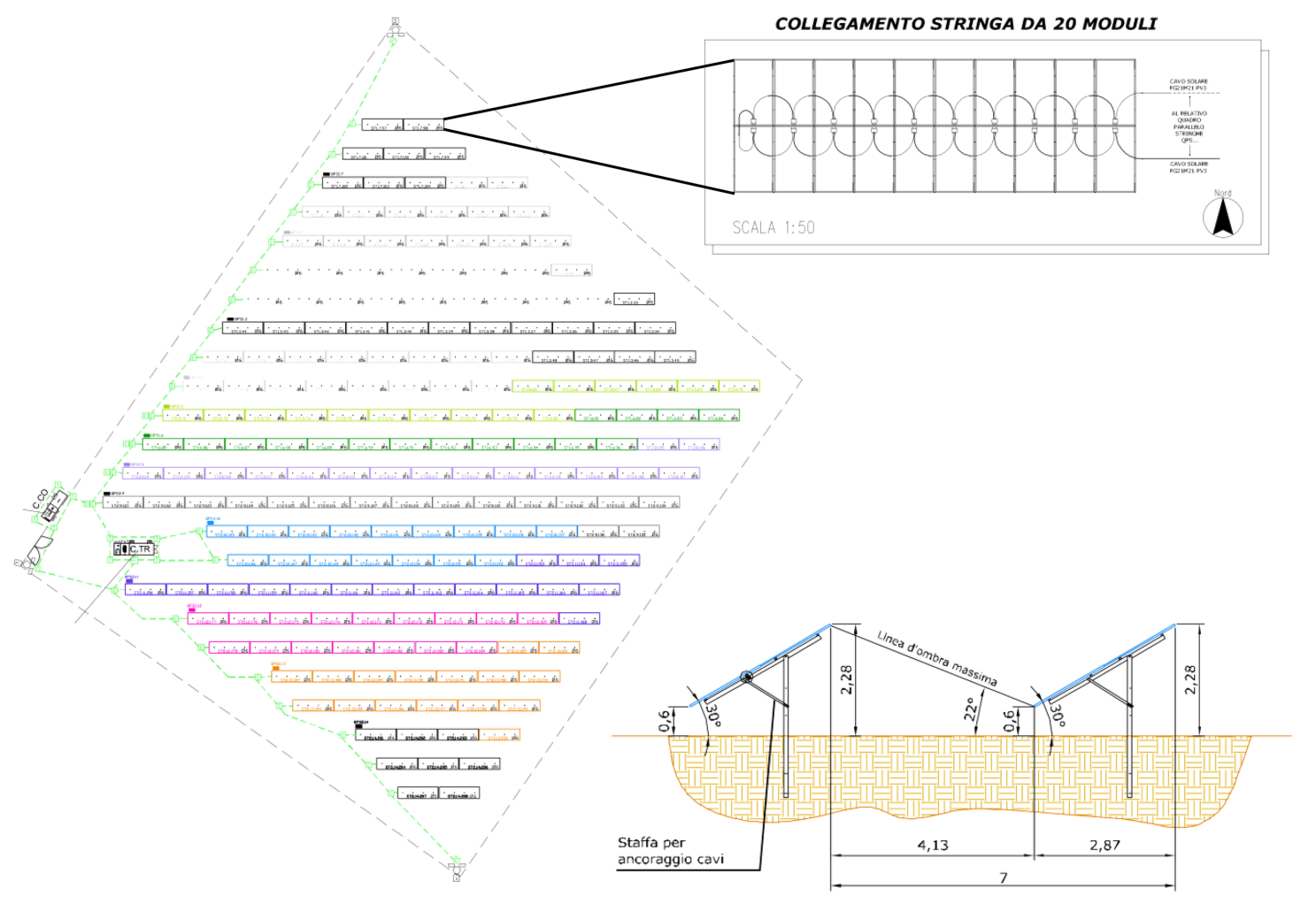

Figure 5. Power plant schematic configuration.

In the evaluation, the three different topologies are modelled in combination with four different MPPT algorithm configurations:

(i) Perturb and Observe;

(ii) Incremental conductance;

(iii) Perturb and Observe with Global Point Tracking subroutine;

(iv) Incremental conductance with Global Point Tracking subroutine.

The data on solar irradiation have been collected from the italian repository on solar irradiation [35]. In the current work, the time resolution of the PV plant simulation is $1 \mathrm{~min}$, although this value can be adjusted in accordance with the MPPT device in use.

For each topology, the best performing MPPT algorithm configuration has been selected and their results have been compared. Among these, the simulated output of the configurations (1) and (2), along with the baseline approach (4), have been used as input data to energy managers of the residential environment.

\subsection{Power Plant Simulation Results}

In the previous work [17], focused on simulating the effect of partial shading on a photovoltaic power plant, the evaluation, based on different weather conditions, has highlighted that, with respect to a micro-block based plant (3), the energy yield losses of a macro-block based plant (2), amount to about $5 \%$, under sunny or cloudy conditions, but can grow up to $20 \%$ in a single cloud scenario. 
On the other hand, for a two inverter plant (1), the energy yield losses amount to about $5 \%$ in cloudy conditions, to about $10 \%$ in sunny conditions, but can rise to about $30 \%$ in a single cloud scenario.

Furthermore, although different MPPT strategies have been investigated, for all scenarios and configurations, the performance difference among them is not enough to overcome the performance difference resulting from the plant design. Moreover, on a yearly perspective, the income difference can estimated to amount to about $3200 €$ per year.

Indeed, in a Micro Grid environment, the design of the plant can affect the energy demand of the Micro Grid towards the main grid; however, even the energy management process may be affected. On purpose, the PV simulation model proposed in [17], which includes a realistic model of solar irradiation that varies in intensity in accordance with both the time of the day and the day of the year, and a model of the plant shading, has been used to evaluate the performance of a PV power plant based on the scenario (a), that assumes the irradiation conditions of a sunny day, and the scenario (b), that assumes the irradiation conditions due to a single cloud moves across the plant, thus shadowing part of the plant in the meantime. Although the actual irradiation levels for each of the 208 groups of panels of the plant are different, by averaging across the plant, the effect of the simulated partial shading can be evaluated, and the irradiation profile for both scenarios can be compared in Figure 6 . The average energy yield amounts to $3031 \mathrm{Wh} / \mathrm{m}^{2}$ in the case of the sunny day, whereas it amounts to $2434 \mathrm{Wh} / \mathrm{m}^{2}$.

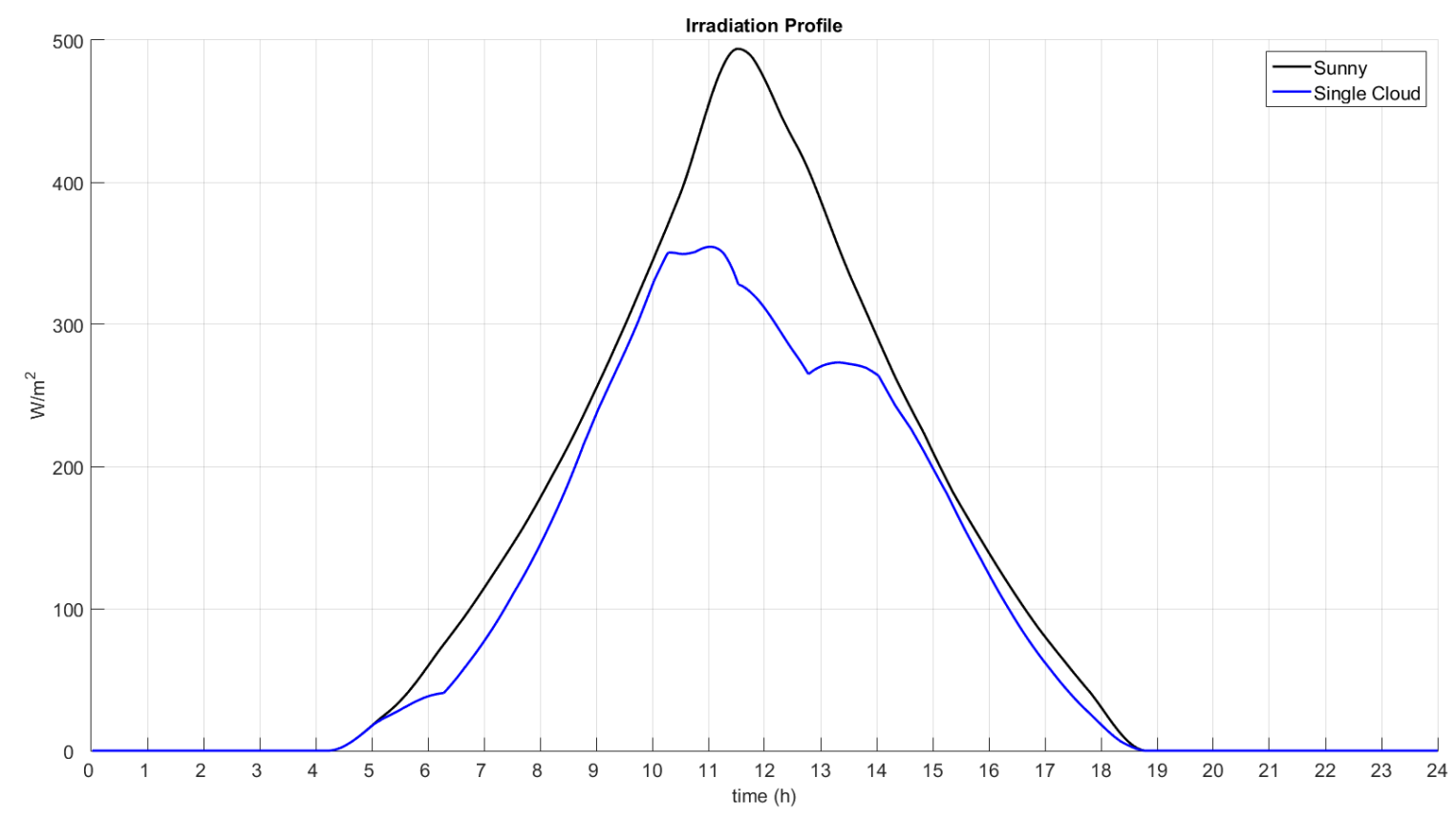

Figure 6. Averaged irradiation level for both scenarios (a) and (b).

Based on the PV simulation model, the power outputs of different configurations have been reported for the sunny day scenario in (Figure 7), whereas the power outputs of the same configurations for the single cloud scenario have been presented in (Figure 8). 


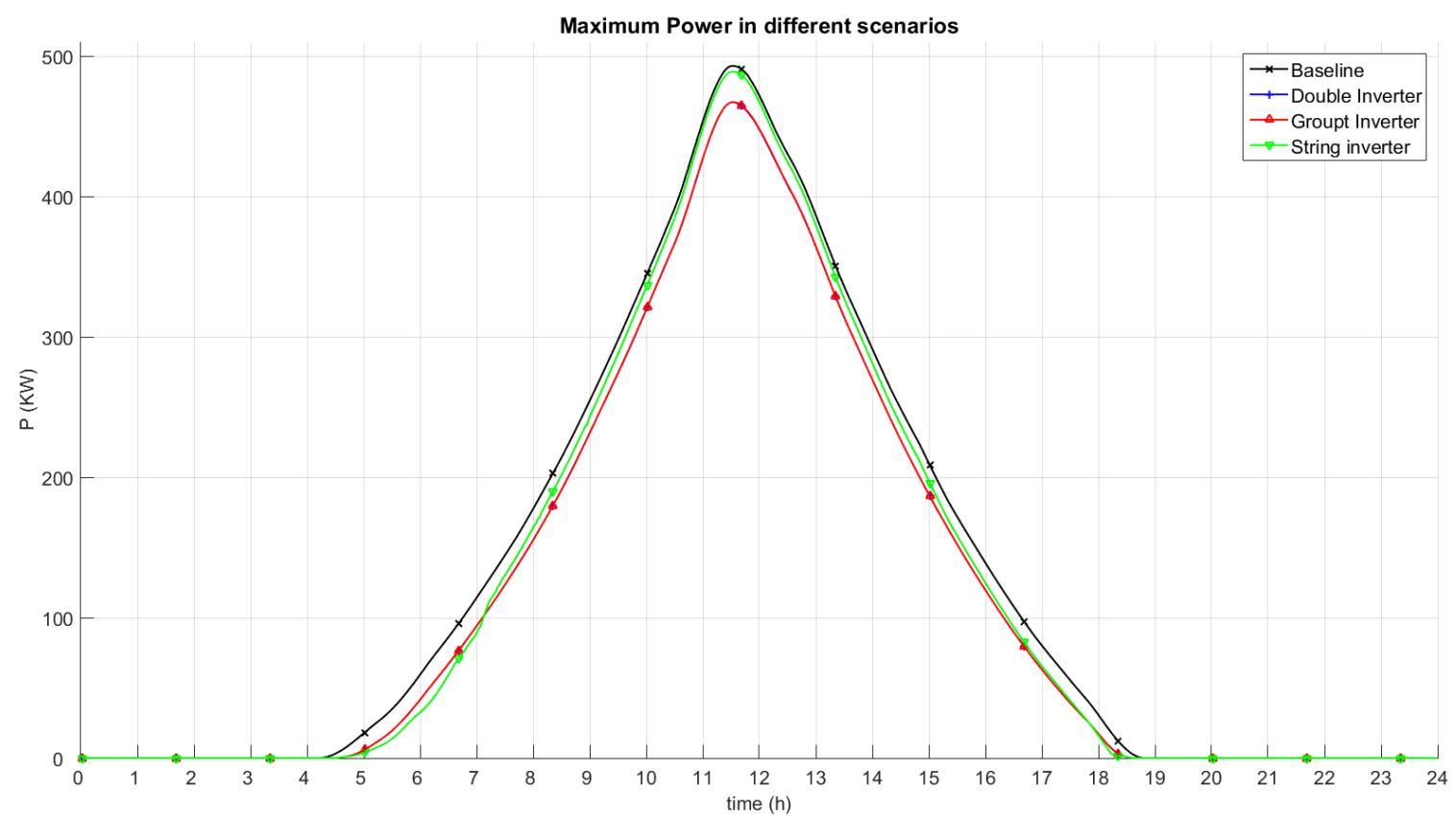

Figure 7. Simulation results for all configurations with scenario (a).

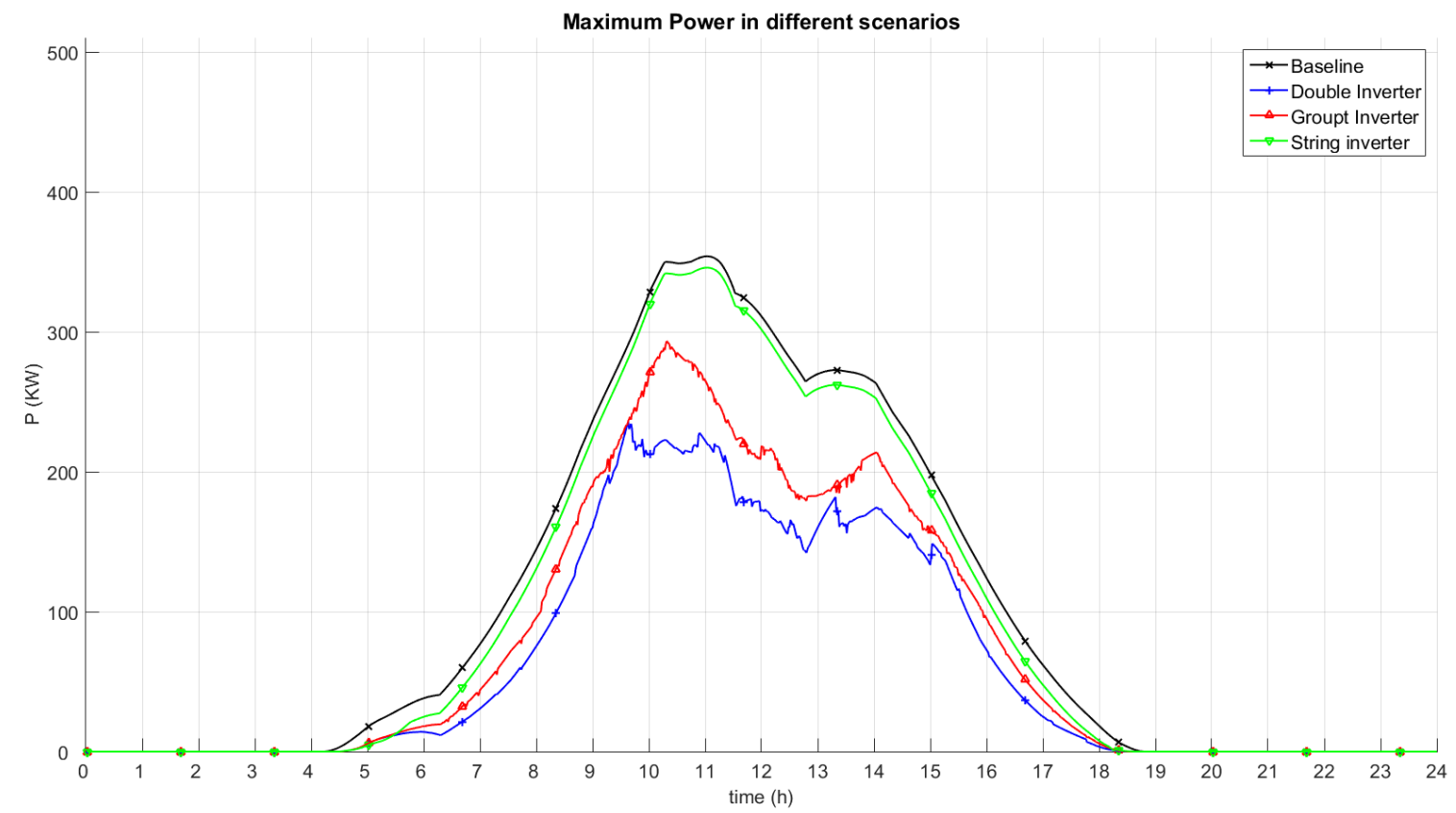

Figure 8. Simulation results for all configurations with scenario (b).

In both scenarios, for each of the PV plant configurations, all four of the MPPT algorithms have been evaluated, and the best performing ones have been compared. For instance, regarding to Configurations (1) and (2), the P\&O paired with the GPT subroutine is the best performing MPPT algorithm. In the case of Configuration (3), the IC MPPT algorithm is the best performer.

As a reference, Configuration (4) is proposed. In this case, the power output is computed by using the irradiation and shading models but assuming a fixed 15\% efficiency level for the whole plant, rather than the PV cell model.

For instance, the value of $15 \%$ corresponds to the peak efficiency of each panel, based on the manufacturer specifications. Since the internal losses of the plant are not accounted for, the baseline 
setup operates the higher overestimation of the power output in both the sunny and the single cloud scenario.

With regards to the most complex topology (3), where each string has its own inverter, since the area of each string is small enough, the irradiation is roughly homogeneous over each string, thus the maximum efficiency can be achieved. Nonetheless, if we compare the result of the Configuration (3) against the baseline counterpart, it can be observed that the difference in power output is higher in the early and late hours, when the lower irradiation levels result in a lower efficiency level. Moreover, this behaviour can be observed in both the sunny and the single cloud configuration.

On the other hand, for both Configurations (1) and (2), the simulated power output overlaps in the case of the sunny day Scenario (Figure 7). Moreover, the difference in power output, with respect to the baseline, reaches its maximum during the central hours of the day.

In the case of the single cloud Scenario (Figure 8), a cloud starts shadowing the plant at about 10 in the morning, and moves completely past the plant at 14; thus, the plant is affected by partial shading in the mean time. In this scenario, Configurations (1) and (2) achieve different results, in accordance with the fact that, with a greater number of inverters, the MPPT algorithm operates in a smaller area, thus improving the performance of the panel output. Therefore, the expected power output for the Configuration (1) is much lower than the expected output of Configuration (2).

Moreover, if the results relating to the single cloud scenario are compared against the results based on the sunny day scenario, it is clear that the performance of the micro-bloc configuration is barely affected by partial shading. On the other hand, the performance of macro-block topology is much more degraded, and the one of the dual inverter configuration even more so. Thus, partial shading does not simply lead to a lower energy yield due to the lower energy levels received by the panels, but also results in a degraded performance of the panels, due to the non-uniform working conditions of the panels.

In terms of energy yield, the absolute results are reported in Table 1. In the best case scenario, the overestimation of the baseline approach amounts to $6 \%$ of the yield of a multi string configuration in sunny days. In the worst case scenario, the overestimation of the baseline approach amounts to $65 \%$ of the energy yield of a dual inverter configuration if a cloud overshadows the plant.

Table 1. Energy yield of the proposed configurations in the different addressed scenarios.

\begin{tabular}{lcc}
\hline \multirow{2}{*}{ Energy Production } & \multicolumn{2}{c}{ Scenarios } \\
\cline { 2 - 3 } & Sunny Day & Single Cloud \\
\hline Reference Energy Yield $\left(\mathrm{kWh} / \mathrm{m}^{2}\right)$ & 3.03 & 2.43 \\
Double Inverter (MWh) & 2.74 & 1.47 \\
Multi inverter (MWh) & 2.74 & 1.78 \\
Single String Inverter (MWh) & 2.85 & 2.26 \\
Baseline (MWh) & 3.02 & 2.43 \\
\hline
\end{tabular}

In other words, the baseline configuration represents the theoretical optimum in terms of power and energy output, whereas the other configurations describe the actual output of a specific real plant topology. Based on the results, the double inverter configuration is the worst performing and the most affected by partial shading, whereas the micro-block configuration is the closest to the theoretical optimum and it is virtually not affected by partial shading. Nonetheless, it should be noted that, in order to implement Configuration (1), only two inverters are required, whereas Configuration (3) is based on 108 inverters. Therefore, the improved performance of Configuration (3) implies a much higher hardware cost, and, thus, it is not usually a viable solution.

\subsection{Energy Management Simulation Case Study}

The simulation framework of the Micro Grid environment models a district composed of 44 buildings, each based on the "Leaf House" described in Section 3.1. Each building is connected 
to the grid and to the local power plant, and includes its own energy manager, two $5.8 \mathrm{kWh} \mathrm{Li}$-ion batteries and electrical storage, a $20 \mathrm{~m}^{2}$ solar thermal collector, a 1300 litre hot water storage tank, and a $16.6 \mathrm{~kW}$ geothermal heat pump. For instance, each building has three stories, two of them have been provided with electrical storage, and the third one is connected directly to the grid and the photovoltaic plant. The solar thermal collector, the hot water storage and the geothermal heat pump, on the other hand, serve the three apartments.

The energy management framework can be used as management tool as well as a design tool. If input data are provided, the framework computes the schedule of the energy management. On the other hand, by providing the computed management schedule along with the input, the framework simulates the energy management process.

The input data set includes the hourly energy PV production, the hourly energy electrical load, the hourly thermal solar production and the hourly thermal needs of the apartments. In the current work, to focus on the improvement provided by the integration with the photovoltaic plant simulation model, aside from solar production, all the remaining data used as inputs are historical records collected from the reference environment, which have been presented in the previous works [26,37]. The solar production, conversely, has been obtained from the photovoltaic plant simulation as described in the previous section.

For simplicity's sake, the 44 buildings have been considered identical, thus the hourly energy electrical load, the hourly thermal solar production and the hourly thermal needs are the same for all of the buildings.

The baseline configuration energy yield has been used as a reference, since the baseline configuration is commonly used in the literature to compute the energy availability of Photovoltaic Plants. The double inverter configuration energy yield, on the other hand, has been proposed as a test case, due to the fact that double inverter plants result in less hardware costs, and thus are more commonly used, with respect to macro-block and micro-block topologies.

To assess the performance in a design process, a design reference, based on the energy yield of the baseline configuration, is compared against a design example based on the energy yield of the double inverter configuration. In both cases, since the management schedule is computed and its execution is simulated based on the same PV plant model, the two phases of the two design processes are matched.

To evaluate the effect of different PV plant modelling, the energy yield of the baseline configuration is used to compute a management schedule, whereas the energy yield of the double inverter configuration is used to represent the yield of a real power plant and simulate the performance of the environment. In this case, the design process is mismatched.

\subsection{Energy Management Simulation Results}

By means of the power output obtained in the previous simulation (Section 4.2), the hourly energy yield has been computed and used as an input of the energy management simulation of the district. For instance, the baseline configuration and the double inverter configuration have been considered as the ideal case scenario and the real case scenario, respectively.

By comparing the performance resulting from the design simulation, for both scenarios, it is possible to evaluate the effects of the energy production overestimation on the design of a Micro Grid. On the other hand, to investigate the energy management simulation, the energy yield of the baseline configuration has been used to plan the management schedule, whereas the energy yield of the dual inverter configuration has been used to simulate the schedule execution. This simulation allows for evaluating the effects of the energy production overestimation in the everyday activity of the energy manager.

With respect to the design process of a Micro Grid, in a sunny day scenario, the power output is described in Figure 7. In this case, if the baseline configuration model is used, the amount of energy, to be delivered to each building, is expected to be $68.6 \mathrm{kWh}$; therefore, the additional amount of energy, supplied by the main grid, required to satisfy the needs of the users of each building, can be assumed to 
be $10.6 \mathrm{kWh}$, thus the expected energy cost amounts to $1.38 €$. On the other hand, based on the double inverter configuration, the energy expected to be delivered amounts to $62.3 \mathrm{kWh}$, the energy expected to be supplied from the main grid amounts to $11.2 \mathrm{kWh}$, and the resulting energy cost amounts to $1.46 €$.

In other words, in the case of the sunny day scenario, a design based on a baseline configuration model results in an expected cost that is $5.5 \%$ lower than the actual cost.

In a single cloud scenario, the power output is described in Figure 8. If the baseline configuration model is used, at design time, the energy delivered to the building is expected to amount to $55.2 \mathrm{kWh}$; the energy supplied from the main grid, then, is assumed to amount to $11.3 \mathrm{kWh}$, and the resulting energy cost amounts to $1.47 €$. If the double inverter configuration model is used, however, the expected energy to be delivered amounts to $33.4 \mathrm{kWh}$, whereas the amount supplied from the main grid is $15.8 \mathrm{kWh}$, and the estimated energy cost amounts to $2.05 €$.

Therefore, with respect to the case of the single cloud scenario, the use of a baseline configuration produces an estimated cost that is $28.3 \%$ lower than the actual cost. Moreover, based on the baseline configuration model, if a cloud shades the plant, the expected cost increase amounts to $6.5 \%$, whereas, if the analysis is based on a double inverter configuration model, the increase of the expected cost amounts to $40 \%$. Hence, in the design of a Micro Grid, the accuracy of the PV plant model and the ability to account for the effect of partial shading are mandatory to correctly assess not only the area and the topology of the plants, but also the characteristics of the many devices and facilities such as the electricity storage.

To investigate the effect of a simplified model on the design process, a mismatched design approach has been proposed. In this case, the energy schedule is planned by using the energy availability computed by means of the baseline configuration model. The schedule execution, however, is simulated using the energy availability computed by means of the double inverter configuration model, which correctly describes the PV plant being used.

In a sunny day scenario, the schedule is computed expecting that $68.6 \mathrm{kWh}$ of energy will be supplied to each building. The delivered energy, however, amounts to $62.3 \mathrm{kWh}$, whereas the energy supplied from the main grid amounts to $13.1 \mathrm{kWh}$, which results in an energy cost equal to $1.71 €$. In a single cloud scenario, the schedule is computed expecting that $55.2 \mathrm{kWh}$ will be supplied to each building. The delivered energy, in this case, will amount to $33.4 \mathrm{kWh}$; therefore, the energy supplied from the main grid is equal to $18.8 \mathrm{kWh}$ and its cost amounts to $2.49 €$.

Therefore, if a baseline configuration model is used to design a Micro Grid that implements a double inverter plant, the energy cost is actually higher than the value that can be expected from a matched design evaluation. In fact, in a sunny day scenario, the energy cost is $23.9 \%$ higher than the one computed in the baseline configuration matched design, and $17.1 \%$ higher than one computed in the double inverter configuration matched design. In a single cloud scenario, the energy cost is also $69.3 \%$ higher than the cost expected from the baseline configuration matched design, and $21.5 \%$ higher than the cost expected from the double inverter configuration matched design.

If the performance drop is considered, the occurrence of the shading effect causes the energy cost to rise from $1.71 €$ to $2.49 €$, which is equal to an additional $45.6 \%$ of the price of a sunny scenario. Thus, the partial shading affects the performance of the energy manager significantly, especially if the model of the PV plant, including the partial shading itself, is not adequate. This allows us to conclude that the integration of the SW framework, proposed in [17], within the energy manager is very effective in order to maximize the energy saving in Micro Grid scenarios.

The cost values of the energy being purchased from the grid for each building are reported also in Table 2. 
Table 2. Energy estimated costs per building in different scenarios.

\begin{tabular}{lcc}
\hline \multirow{2}{*}{ Design Approach } & \multicolumn{2}{c}{ Scenarios } \\
\cline { 2 - 3 } & Sunny Day & Single Cloud \\
\hline Matched-baseline $(€)$ & 1.38 & 1.47 \\
Matched-double inverter $(€)$ & 1.46 & 2.05 \\
Mismatched $(€)$ & 1.71 & 2.49 \\
\hline
\end{tabular}

\section{Conclusions}

In the current work, a software framework that models the behaviour of a real life photovoltaic power plant has been integrated with a software framework, aimed at energy management in a Micro Grid environment, with the objective of investigating the effect of accurate energy production estimation in both the design and the management processes of a Micro Grid environment. The software is also made available upon request.

The PV plant simulation model is based on a real-life PV power plant that operates based on the behaviour of the PN junctions within the panels, the effect of MPPT based inverters, the partial shading as well as the elevation angle of the sun and the tilt angle of the panels. The energy management simulation model is also based on a real-life Micro Grid environment and operates by computing the energy flux within the systems of the environment. Thus, the flux of both electrical and thermal energy among the devices in the house are used to schedule the energy management activity. Since the energy management framework is suitable for both design and management, the addition of a PV plant simulation model further enhances the accuracy of cost estimations, energy needs and building performance.

In fact, with respect to the accurate PV modelling, a simplified model leads to the overestimation of the energy availability. For instance, the experiments reveal that an estimation based on a simplified PV model can exceed by $65 \%$ the actual production. This can affect both design and management of a Micro Grid, since it leads to an underestimation of energy needs and costs.

In particular, in sunny days, the expected cost based on a simplified PV model can be about $5.5 \%$ lower than the cost based on the double inverter model. In single cloud scenarios, however, the underrating can reach $28.3 \%$. The same holds for the management process. In fact, if the expected energy yield is estimated by means of a simplified PV model, the actual cost can be from $17.1 \%$ to $21.5 \%$ higher than the theoretical cost expected at design time. The obtained results show that the proposed software framework can be employed as a useful design tool for properly sizing the power plant as well as for improving the performance of the energy manager.

Although the results highlight the benefits of an accurate PV plant model, further enhancement and development are required, in order to better integrate the PV model in the energy management framework. On purpose, an evaluation based on a full fledged design software such as the System Advisor Model provided by NREL will also be carried out. Moreover, the support from weather stations as well as an improved modelling of the shading may be required to increase the accuracy of the energy yield prediction. To better analyse the issue, however, weather forecasts as well as weather historical data may be needed to evaluate the energy management process in a proper fashion.

Acknowledgments: Authors were supported by the Italian National Technology Cluster project SHELL (Shared interoperable Home Ecosystems for a green, comfortabLe and safe Living), project code: CTN01 00128111357.

Author Contributions: The authors contributed in the same way for the entirety of the paper.

Conflicts of Interest: The authors declare no conflict of interest. 


\section{References}

1. Bernstein, P.A.; Heuer, M.; Wenske, M. Fuel cell system as a part of the smart grid. In Proceedings of the IEEE Grenoble Conference, Grenoble, France, 16-20 June 2013; pp. 1-4.

2. Tomal, M.U.; Gabbar, H.A. Key performance assessment of fuel cell based distributed energy generation system in resilient micro energy grid. In Proceedings of the IEEE International Conference on Smart Energy Grid Engineering (SEGE), Toronto, ON, Canada, 17-19 August 2015; pp. 1-6.

3. Sauhats, A.; Linkevics, O.; Varfolomejeva, R.; Zalostiba, D.; Kunickis, M.; Balodis, M. Towards smart control and optimization of the small-scale power system. In Proceedings of the IEEE 5th International Conference on Power Engineering, Energy and Electrical Drives (POWERENG), Riga, Latvia, 11-13 May 2015; pp. 440-446.

4. Chen, P.; Jiang, Z.; Tang, Z.; Guan, L. Effect of electricity feed-in tariff on the planning of CCHP Micro-systems. In Proceedings of the IEEE PES Asia-Pacific Power and Energy Engineering Conference (APPEEC), Xi'an, China, 25-28 October 2016; pp. 875-879.

5. Colak, I.; Ayaz, M.S.; Bilgili, M.; Boran, K. Cost benefit analysis of wind turbines in smart grid systems. In Proceedings of the 16th International Power Electronics and Motion Control Conference and Exposition, Antalya, Turkey, 17-19 September 2014; pp. 1295-1299.

6. Abo-Al-Ez, K.M.; Tzoneva, R. Modelling and adaptive control of a wind turbine for smart grid applications. In Proceedings of the International Conference on the Industrial and Commercial Use of Energy (ICUE), Cape Town, South Africa, 18-19 August 2015; pp. 332-339.

7. Tamalouzt, S.; Benyahia, N.; Rekioua, T.; Abdessemed, R. A Doubly Fed induction generator wind turbine and fuel cell hybrid power sources system for micro-grid applications. In Proceedings of the 3rd International Conference on Control, Engineering Information Technology (CEIT), Tlemcen, Algeria, 25-27 May 2015; pp. 1-6.

8. Patel, H.; Agarwal, V. MATLAB-based modeling to study the effects of partial shading on PV array characteristics. IEEE Trans. Energy Convers. 2008, 23, 302-310.

9. Khatoon, S.; Ibraheem; Jalil, M.F. Analysis of solar photovoltaic array under partial shading conditions for different array configrations. In Proceedings of the 2014 Innovative Applications of Computational Intelligence on Power, Energy and Controls with their impact on Humanity (CIPECH), Ghaziabad, India, 28-29 November 2014; pp. 452-456.

10. Maki, A.; Valkealahti, S. Effect of photovoltaic generator components on the number of MPPs under partial shading conditions. IEEE Trans. Energy Convers. 2013, 28, 1008-1017.

11. Dolara, A.; Lazaroiu, G.C.; Leva, S.; Manzolini, G. Experimental investigation of partial shading scenarios on PV (photovoltaic) modules. Energy 2013, 55, 466-475.

12. Jena, D.; Ramana, V.V. Simple and accurate method of modeling Photovoltaic module: A different approach. In Proceedings of the 2013 International Conference on Green Computing, Communication and Conservation of Energy (ICGCE), Chennai, India, 12-14 December 2013; pp. 465-469.

13. Aashoor, F.; Robinson, F. A variable step size perturb and observe algorithm for photovoltaic maximum power point tracking. In Proceedings of the 2012 47th International Universities Power Engineering Conference (UPEC), London, UK, 4-7 September 2012; pp. 1-6.

14. Sera, D.; Mathe, L.; Kerekes, T.; Spataru, S.V.; Teodorescu, R. On the perturb-and-observe and incremental conductance MPPT methods for PV systems. IEEE J. Photovolt. 2013, 3, 1070-1078.

15. Wasynezuk, O. Dynamic behavior of a class of photovoltaic power systems. IEEE Trans. Power Appl. Syst. 1983, PAS-102, 3031-3037.

16. Kazan, F.; Karaki, S.; Jabr, R.A.; Mansour, M. Maximum power point tracking using ripple correlation and incremental conductance. In Proceedings of the 2012 47th International Universities Power Engineering Conference (UPEC), London, UK, 4-7 September 2012; pp. 1-6.

17. Severini, M.; Scorrano, A.; Squartini, S.; Fagiani, M.; Piazza, F. SW framework for simulation and evaluation of partial shading effects in configurable PV systems. In Proceedings of the IEEE 16th International Conference on Environment and Electrical Engineering (EEEIC), Florence, Italy, 7-10 June 2016; pp. 1-6.

18. Huang, K.; Wang, X.; Wang, L. Optimal energy management of grid-connected photovoltaic micro-grid. In Proceedings of the IEEE International Conference on Cyber Technology in Automation, Control, and Intelligent Systems (CYBER), Shenyang, China, 8-12 June 2015; pp. 234-239. 
19. Leva, S.; Dolara, A.; Grimaccia, F.; Mussetta, M.; Ogliari, E. Analysis and validation of $24 \mathrm{~h}$ ahead neural network forecasting of photovoltaic output power. Math. Comput. Simul. 2017, 131, 88-100.

20. Cameron, C.P.; Boyson, W.E.; Riley, D.M. Comparison of PV system performance-model predictions with measured PV system performance. In Proceedings of the 2008 33rd IEEE Photovoltaic Specialists Conference, San Diego, CA, USA, 11-16 May 2008; pp. 1-6.

21. Hua, C.; Lin, J.; Shen, C. Implementation of a DSP-controlled photovoltaic system with peak power tracking. IEEE Trans. Ind. Electron. 1998, 45, 99-107.

22. Kish, G.J.; Lee, J.J.; Lehn, P. Modelling and control of photovoltaic panels utilising the incremental conductance method for maximum power point tracking. Renew. Power Gener. IET 2012, 6, 259-266.

23. Xiong, Y.; Qian, S.; Xu, J. Research on constant voltage with incremental conductance MPPT method. In Proceedings of the 2012 Asia-Pacific Power and Energy Engineering Conference (APPEEC), Shanghai, China, 27-29 March 2012; pp. 1-4.

24. Shah, K.B.; Joshi, L.P. Comparative analysis of incremental conductance base MPPT for multi-string photovoltaic system. In Proceedings of the 2013 Nirma University International Conference on Engineering (NUiCONE), Ahmedabad, India, 28-30 November 2013; pp. 1-6.

25. Patel, H.; Agarwal, V. Maximum power point tracking scheme for PV systems operating under partially shaded conditions. IEEE Trans. Ind. Electron. 2008, 55, 1689-1698.

26. Comodi, G.; Giantomassi, A.; Severini, M.; Squartini, S.; Ferracuti, F.; Fonti, A.; Cesarini, D.N.; Morodo, M.; Polonara, F. Multi-apartment residential microgrid with electrical and thermal storage devices: Experimental analysis and simulation of energy management strategies. Appl. Energy 2015, 137, 854-866.

27. Severini, M.; Squartini, S.; Piazza, F. Hybrid soft computing algorithmic framework for smart home energy management. Soft Comput. 2013, 17, 1983-2005.

28. Severini, M.; Squartini, S.; Piazza, F. Computational framework based on task and resource scheduling for micro grid design. In Proceedings of the 2014 International Joint Conference on Neural Networks (IJCNN), Beijing, China, 6-11 July 2014; pp. 1695-1702.

29. Tamrakar, V.; Gupta, S.C.; Sawle, Y. Study of characteristics of single and double diode electrical equivalent circuit models of solar PV module. In Proceedings of the 2015 International Conference on Energy Systems and Applications, Pune, India, 30 October-1 November 2015; pp. 312-317.

30. Sah, C.T.; Noyce, R.; Shockley, W. Carrier generation and recombination in pn junctions and pn junction characteristics. Proc. IRE 1957, 45, 1228-1243.

31. Tsai, H.L.; Tu, C.S.; Su, Y.J. Development of generalized photovoltaic model using MATLAB/SIMULINK. In Proceedings of the World Congress on Engineering and Computer Science, San Francisco, CA, USA, 22-24 October 2008; Volume 2008, pp. 1-6.

32. Quaschning, V.; Hanitsch, R. Numerical simulation of current-voltage characteristics of photovoltaic systems with shaded solar cells. Sol. Energy 1996, 56, 513-520.

33. Ishaque, K.; Salam, Z.; Syafaruddin. A comprehensive MATLAB Simulink PV system simulator with partial shading capability based on two-diode model. Sol. Energy 2011, 85, 2217-2227.

34. Ishaque, K.; Salam, Z.; Taheri, H. Simple, fast and accurate two-diode model for photovoltaic modules. Sol. Energy Mater. Sol. Cells 2011, 95, 586-594.

35. Rinnovabili, E.F. Atlante Italiano della Radiazione Solare. Available online: http:/ /www.solaritaly.enea.it/ (accessed on 15 February 2015).

36. Cellura, M.; Ciulla, G.; Lo Brano, V.; Orioli, A.; Campanella, L.; Guarino, F.; Cesarini, D.N. The Redesign of an Italian Building to Reach Net Zero Energy Performances: A Case Study of the SHC Task 40-ECBCS Annex 52. ASHRAE Trans. 2011, 117, 331-339.

37. Severini, M.; Squartini, S.; Fagiani, M.; Piazza, F. Energy management with the support of dynamic pricing strategies in real micro-grid scenarios. In Proceedings of the 2015 International Joint Conference on Neural Networks (IJCNN), Killarney, Ireland, 12-17 July 2015; pp. 1-8.

38. Currie, J.; Wilson, D.I. OPTI: Lowering the Barrier Between Open Source Optimizers and the Industrial MATLAB User. In Foundations of Computer-Aided Process Operations; SaSahinidis, N., Pinto, J., Eds.; Elsevier Science Ltd.: Toronto, ON, Canada, 2012.

39. CanadianSolar. Available online: http://www.solarelectricsupply.com/canadian-solar-cs6p-240-solarpanels-110 (accessed on 15 February 2015). 
40. Zheng, H.; Li, S.; Challoo, R.; Proano, J. Shading and bypass diode impacts to energy extraction of PV arrays under different converter configurations. Renew. Energy 2014, 68, 58-66.

41. Diaz-Dorado, E.; Suarez-Garcia, A.; Carrillo, C.; Cidras, J. Influence of the shadows in photovoltaic systems with different configurations of bypass diodes. In Proceedings of the SPEEDAM 2010, Pisa, Italy, 14-16 June 2010; pp. 134-139.

42. Ramaprabha, R.; Mathur, B. A comprehensive review and analysis of solar photovoltaic array configurations under partial shaded conditions. Int. J. Photoenergy 2012, 2012, 120214.

(c) 